\title{
Technical Data Extraction and Representation in Expert CAPP System
}

\author{
Rafik Elaakil, Rechia Ahmed, Abdelilah El Mesbahi, Oussama Jaider \\ Department of Mechanical Engineering, Faculty of Sciences and Technics of Tangier, \\ Ancienne Route de l'Aéroport, Km 10, Ziaten. BP : 416. Tanger - Maroc \\ rafik.elaakil@gmail.com,rechia@hotmail.com
}

\begin{abstract}
Computer-aided process planning (CAPP) is an essential interface for linking design and manufacturing processes, the purpose of CAPP is to transform a part design specification obtained from CAD system into a sequence of machining operations which can be used by CAM system involving the selection of necessary manufacturing processes in order to transform a designed part into a final part economically and competitively. This paper focus on the extraction of tolerances (GD\&T) and their association to the related features embedded into an expert CAPP system.
\end{abstract}

Keywords: CAD/CAM, CAPP, machining operation sequencing, Expert system, knowledge bases, Artificial intelligence

\section{Introduction}

Computer Aided Process Planning is an important activity in an intelligent manufacturing system. Several techniques have been proposed, implemented and tested in the context of generative CAPP; it consists of several activities including machining feature recognition, selection of machining operation type, selection of manufacturing resource, setup planning and operation sequencing.

The objective of precedence-constrained sequencing problem is to locate the optimal sequence dealing with time and costs, so that the resulting operation sequence could satisfy those constraints established by geometric technological, economical requirements, and then generate the optimal or near optimal operation sequences.

The extraction of tolerances data is an essential key that help to identify the basic constraints of the related input part. To achieve a complete representation for a process-planning model, the tolerances data (GD\&T) needs to be completely extracted and represented through the input data file.

The development of a robust methodology for extracting this data for usea knowledge-based expert system, incorporating an artificial intelligence algorithm is the main thrust of the work reported in this paper. 


\section{Related researches work and review}

Form features are not sufficient so that the part will be manufactured at a reasonable cost and will perform its intended function. Most researchers have concentrated on geometric data extraction, [1] describes a method for the generation of tolerance specifications from product data, for each feature one or more geometric controls will have to be identified, [2]presents a way to interpret the Geometric Dimensioning and Tolerancing (GD\&T) specifications in STEP for tolerance analysis by utilizing OntoSTEP plugin [3] which consists of translating the APs defined in the EXPRESS language [4], and the actual instance files represented using the ISO 10303-21 (or simply part21)format [5].

[6] presents an on-machine measurement data model based on STEP-NC, this model represents tasks in process plan in an object-oriented, containing information on geometry and tolerances.

\section{ICAPP-TURN Architecture}

A main objective in modern design and advanced machining process is to achieve a significant reduction in product lead time through seamless integration between the various design and process planning activities. The concept of using component features to integrate a design system and a manufacturing system has been a major research direction in recent year, [7] describes the characteristics of an existing expert systems building tools and discusses the use and the importance of these basic characteristics from a perspective of computer aided process planning (CAPP) requirements in order to automate the process planning functions.

The framework of the proposed system is decomposed into several modules, Figure 1 show the conceptual model that defines the structure of ICAPP-TURN.

The process start from the knowledge acquisition step that consists of structuring and organizing knowledge from one source, usually human experts, so it can be used and represented into the knowledge representation module.

The knowledge database represents a centralized repository to store constraints and rules provided by the knowledge representation module, in other side, the technical data information is retrieved through the integration module that extract the technical information of such part, which can be represented to the system across the data representation module that maps this information into technical objects.

The core system is represented as an inference engine which helps to determine the optimal sequences plan using an

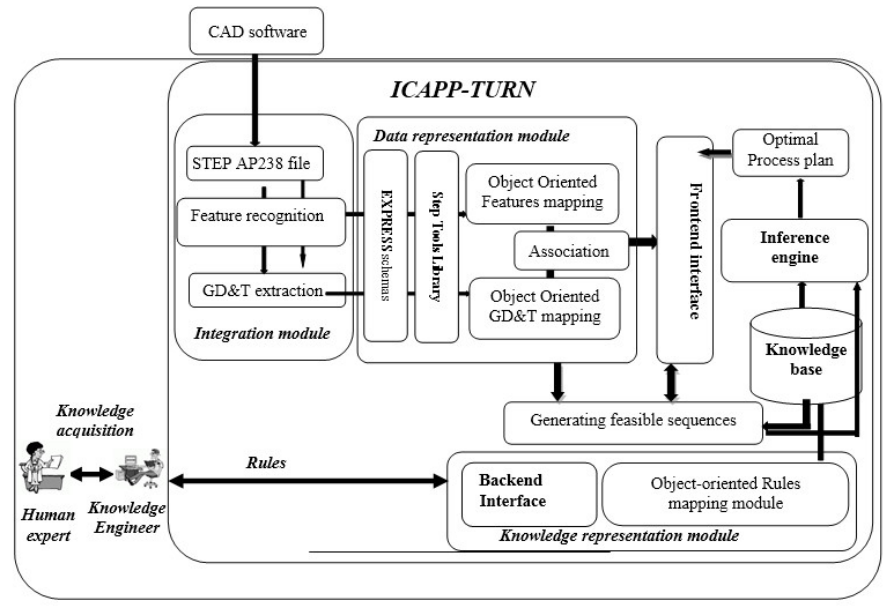


Artificial intelligence method, this module interacts with the Knowledge database.

\section{Technical Data Integration and Representation modules}

The integration module consists of recognizing manufacturing features and tolerances from a STEP AP238 file, then associate each tolerance to the related feature through the data representation module.

[8] has developed an automatic feature recognition system for rotational parts which adopted a STEP AP203 file as input to the system.

STEP AP203 is an application protocol of the Standard for the Exchange of Product model data (STEP), defined as the international standard ISO 10303 [9]. For the fact that the definitions of geometric and topological entities of the STEP AP203 are included in the AP238, we have used this feature recognition system to detect features from a STEP AP238, generated by STEP-NC Machine[ ]by applying this recognition, the system can recognize multiple features types. Figure 2 show an input part to our system. The feature recognition system recognizes in this case one shoulder, which is composed by 3 advanced face, one planar surface and two cylindrical surfaces:

\#235=ADVANCED_FACE('Corps principal',(\#171,\#175),\#179,.T.);

\#47=ADVANCED_FACE('Corps principal',(\#48),\#82,.T.);

\#87=ADVANCED_FACE('Corps principal',(\#88),\#82,.T.);

In the following section, we will explain how a type of tolerance such as surface roughness (named as SURFACE_TEXTURE_PARAMETER) is extracted from STEP AP238 file, and then associated to the corresponding feature.

As shown in Fig. 2, the face with the attribute $\# 235$ has a roughness of 1,6um as a value; this tolerance is embedded in the STEP AP238 data file of the example part asshown in Figure 3.

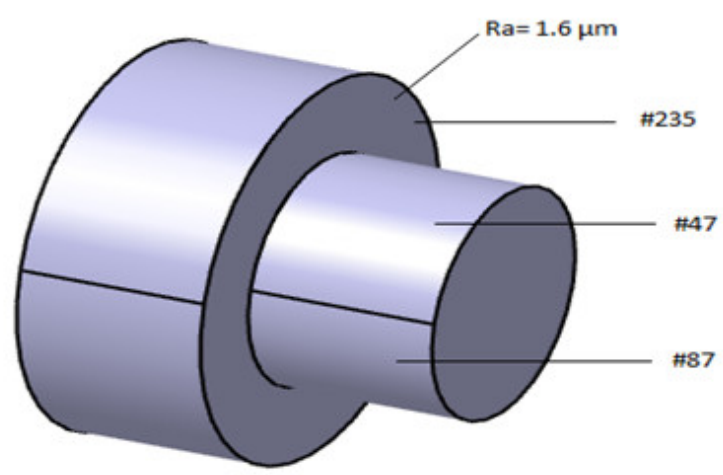

Figure2: An input example part that contains a toleranced feature 


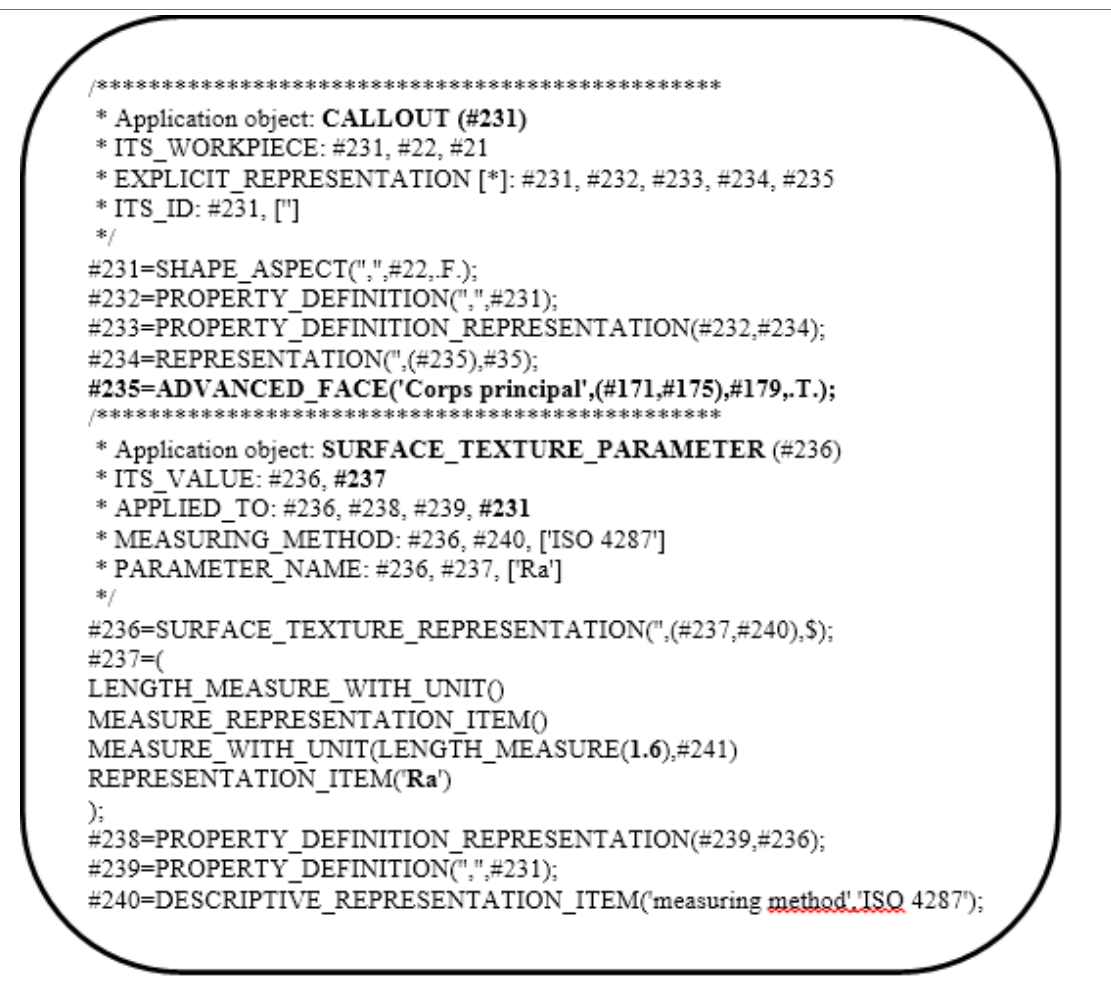

Figure 3: A partial STEP AP238 text file of the definition of roughness of the part of Fig.2

In figure 3, the CALLOUT ( ) entity defines a callout identifying a particular face (ADVANCED_FACE) on the 


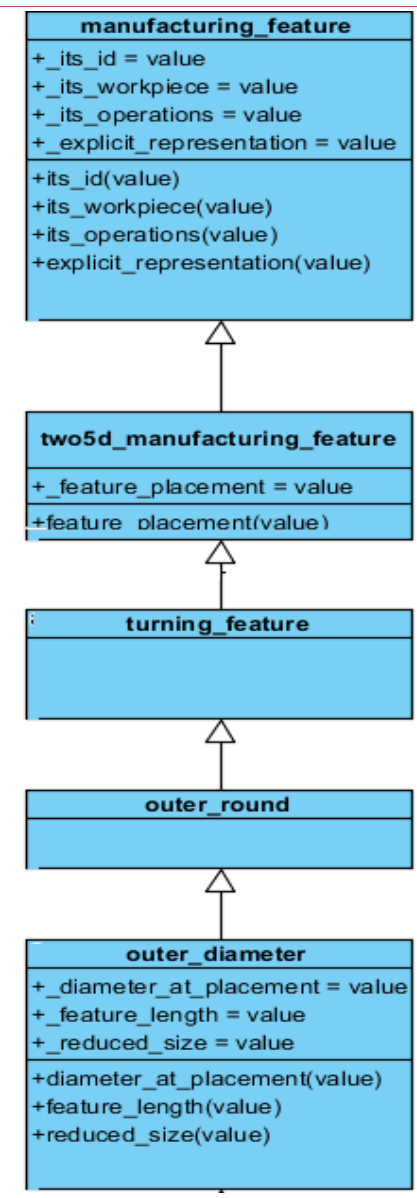

Figure 4: The UML diagram of the class outer_diameter

part that can have some properties (e.g. surface roughness). The SURFACE_TEXTURE_PARAMETERentity defines surface roughness to one or more faces; it has an attribute denoted as ITS_VALUE: \#236, \#237, where \#237 is a pointer to the value and the type of surface roughness. The SURFACE_TEXTURE_PARAMETER ( ) entity has a second attribute denoted as APPLIED_TO: \#236, \#238, \#239, \#231, where \#231 is a pointer to the callout of \#231.

This means that the SURFACE_TEXTURE_PARAMETER is applied to the ADVANCED_FACE with the pointer \#235.

Thus, following these indicators brings us allocate the surface roughness, its value and the advanced face to which thisroughness is applied using definedfunctions. The feature recognition module recognizes each feature and represents it as an object, for which The ADVANCED_FACE (that compose the feature) are known. This leads us to specify the feature for which the roughness has been applied to, however there is no association between the feature and the tolerance in term of objects. In fact, STEP Tools Library used in this module[11], generate an oriented object classes based on EXPRESS schema; the outer_diameter class doesn't have an attribute representing the tolerance applied to the concerned feature.

Figure 4 shows the class representing the outer_diameter feature according to ISO 14649, and the upstream classes from which the outer_diameter class inherits attributes. 
As shown in Fig.4, the outer_diameter class doesn't have an attribute that represents the tolerance applied to the concerned feature. The solution proposed in this paper concerns the creation of an association between the parent class ofthe recognized features and the classes representing the tolerance, which means that an instance of the class outer_diameter for example is composed of zero or a list of instances (zero-to-Many Mapping) for each type of tolerance, whether it is a surface_texture_parameter or another tolerance type defined in STEP AP238.

Figure 5 shows the new association between turning_feature parent class and the new classes that can represent any type of tolerance. Taking the fact that tolerances classes have become part of the turning_feature class, each manufacturing feature (for example the outer_diameter class) for which the turning_feature class is parent will inherits tolerances as objects.

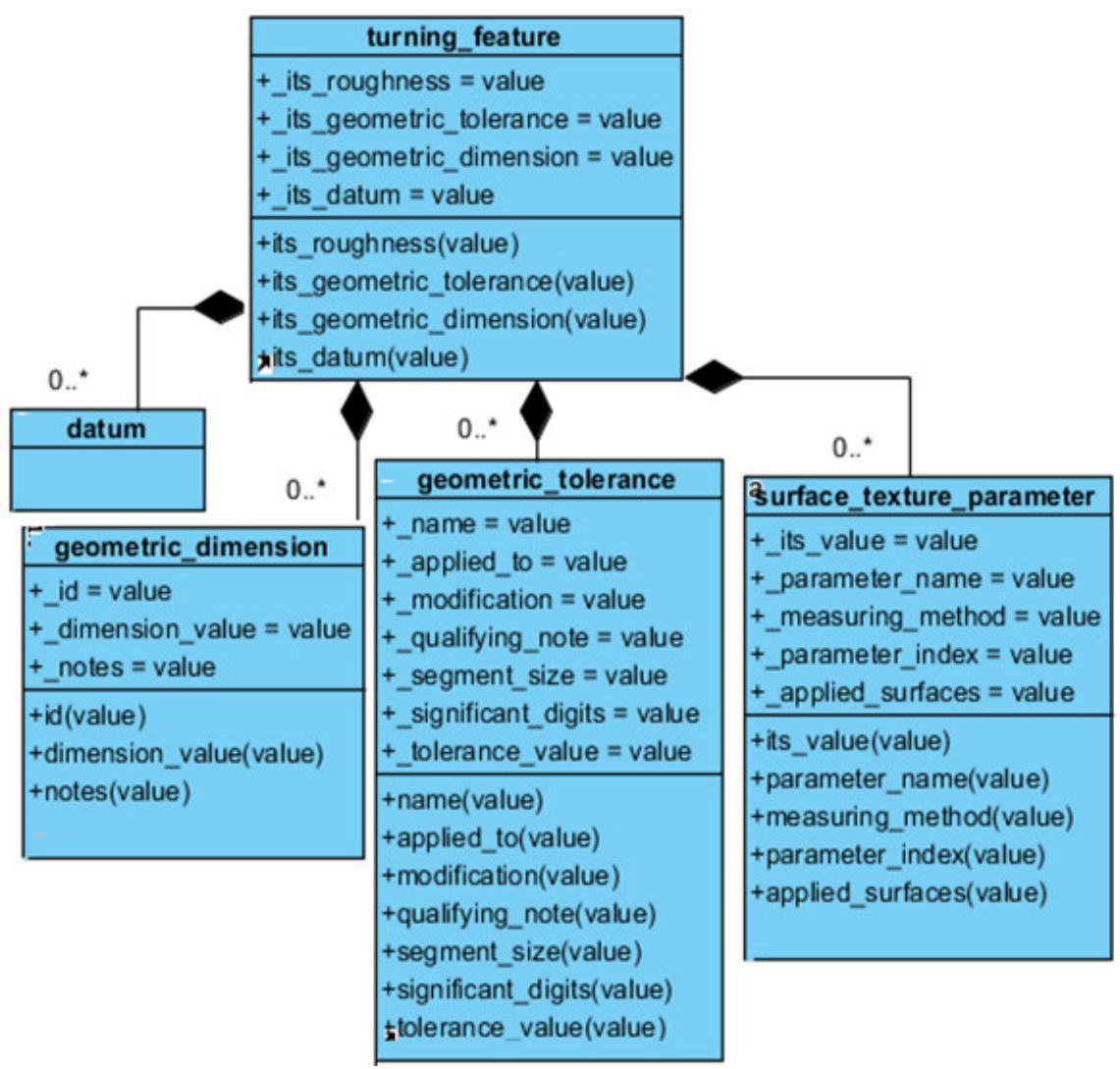

Figure 5: New association between turning feature and related tolerances

After describing how surface roughness is embedded in the STEP AP238 data file and how tolerances can be associated to the related manufacturing features, the parameters representing tolerances will be searched and located using defined methods.

Figure 6 show the class outer_diameter containing the concerned tolerances inherited from the turning_featureparent class.

It must be noticed that surface_texture_parameter has been taken just an example for its simplicity; the new inherited classes can also handle other types of tolerances defined in STEP AP238 such as, geometric tolerances, geometric dimension and Datum. 
Rafik Elaakil, Rechia Ahmed, Abdelilah El Mesbahi, Oussama Jaider; Technical Data Extraction and Representation

in Expert CAPP System. Transactions on Machine Learning and Artificial Intelligence, Special Issue Vol 5 No 4 August (2017); pp: 66-74

The input file that has been taken in this example will just consider the roughness since it is the only tolerance that has been applied to this feature. Other tolerances types won't be instantiated.

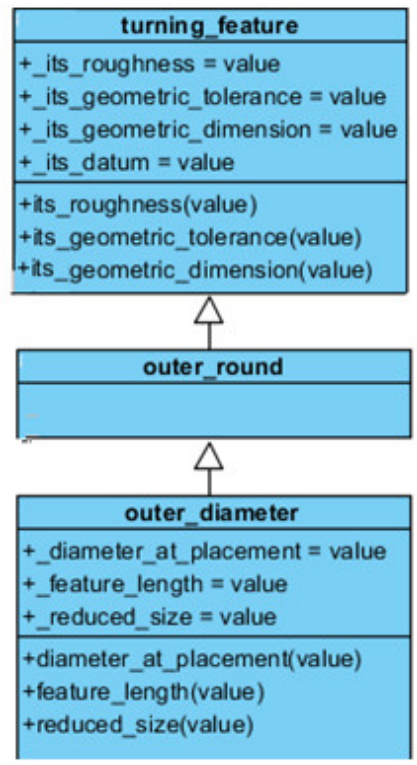

Figure 6: The new association representing the outer_diameter class that inherit the tolerances type

Therefore, the main tasks in GD\&T extraction and features/tolerances association are to extract, instantiate then associate between each manufacturing feature and its related tolerance as an attribute.

Figure 7 illustrates the flowchart of the algorithm established for the surface_texture_parameter extraction from STEP AP238, and its association with the corresponding feature.

For the positional roughness, the algorithm is divided into a three-stage process.

In the first stage, the callout object and the positional SURFACE_TEXTURE_PARAMETERare located.

The second stage consists in instantiating a new object of type surface_texture_parameter. The last stage consists in associating the surface_texture_parameter to the corresponding manufacturing feature.

Taking the fact that the ADVANCED_FACEs that compose each feature are known, the new tolerances objects will be then associated to the corresponding feature by applying the new association method explained in this work.

That solution will lead us to get objects inherited from turning_feature, representing tolerances related to the corresponding manufacturing features. 


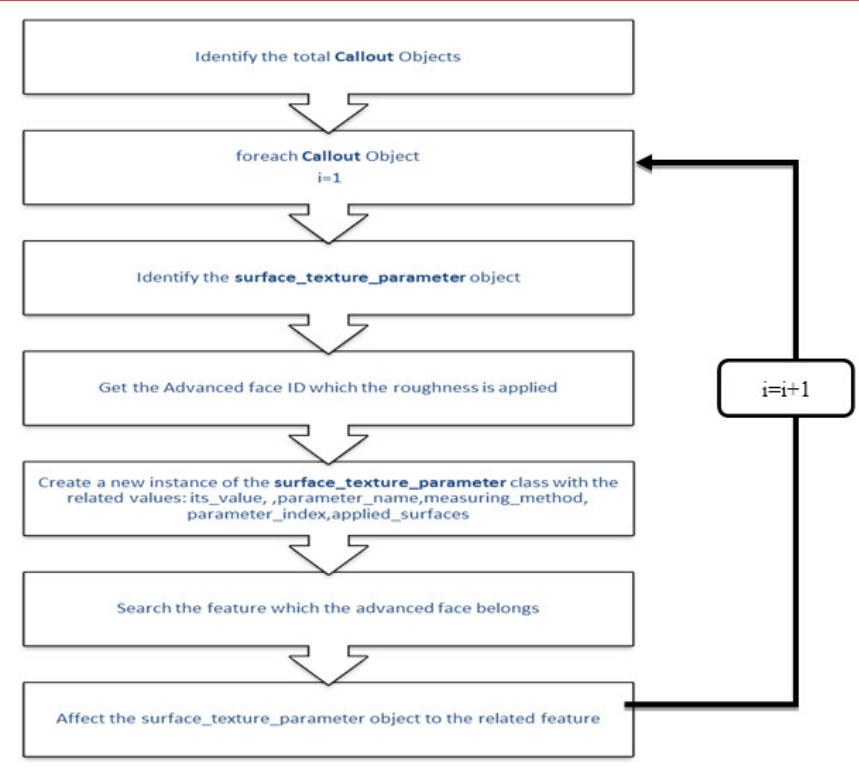

Figure 7: Flowchart of the association between the surface_texture_parameter object and its related manufacturing feature

\section{Conclusion}

In this paper, we have developed a new approach to extract tolerances from a step ap238 file, and then associate them with their related machining features. An algorithm was implemented and embedded into an expert system called ICAPP-TURNto extract and map tolerances data for process planning. The main task is to locate each tolerance and associate it with the related feature.

The data integration module described in this paper contains the GD\&T extraction methods from a step ap238 data input file. These methods locate the surface roughness, its value and the advanced face to which the roughness is applied using custom defined functions. The data representation module creates the mapping of the related machining features and tolerances to the oriented objects, then makes associations between these objects based on a new approach for conception and implementation of object oriented that improves the reusability, flexibility and the relationships between the machining features and their tolerances as properties. These modules represent an input data for ICAPP-TURN system.

Thus, in a future work, we will describe the knowledge representation module which provides the knowledge to the centralized database. This module receives the input knowledge about rules and constraints, and then represents this information in a form that ICAAP-Turn can utilize to store data and resolve tasks.

\section{REFERENCES}

[1] Antonio Armillotta,A method for computer-aided specification of geometric tolerances,Dipartimento di Meccanica, Politecnico di Milano, ViaLa Masa 1, 20156 Milano, Italy, Computer-Aided Design 45 (2013) 1604-1616. 
Rafik Elaakil, Rechia Ahmed, Abdelilah El Mesbahi, Oussama Jaider; Technical Data Extraction and Representation

in Expert CAPP System. Transactions on Machine Learning and Artificial Intelligence, Special Issue Vol 5 No 4 August (2017); pp: 66-74

[2] Mehmet I. Sarigecili , Utpal Roya,Interpreting the semantics of GD\&T specifications of a product for tolerance analysis, Computer-Aided Design 47 (2014) 72-84.

[3] OntoSTEP Plug-in. July, 2012. http://www.nist.gov/el/msid/ontostep.cfm.

[4] ISO_10303-11, Industrial automation systems and integration - Product data representation and exchange -Part 11: Description methods: The EXPRESS language.

[5] ISO_10303-21, Industrial automation systems andintegration - Product data representationand exchange -Part 21: Implementation methods: Clear textencoding of the exchange structure.

[6] Pei Lei, Lianyu Zheng,A STEP-NC compatible on-machine measurement system with automated correlation of inspection data. Applied Mechanics and Materials Vols. 397-400 (2013) pp 1772-1777

[7] HakkiEskicioglu, The Use of Expert System Building Tools in Process Planning, Middle East Technical University, Ankara, 1992, Engineering Applications of Artificial Intelligence.Vol. 5, No. 1, pp. 33-42, 1992.

[8] JaiderOussama, al. Int. Journal of Engineering Research and Applications ISSN : 2248-9622, Vol. 4, Issue 5( Version 1), May 2014

[9] STEP Application Protocol (AP) 203 editions 1 \& 2,Configuration Controlled 3D Designs of Mechanical Partsand Assemblies, ISO10303 1994, (2007) \& (2009).

[10] http://step-nc-machine.software.informer.com/9.1/

[11] http://www.steptools.com/ 